\title{
Interleukin 17A Promotes Cell Migration, Enhances anoikis resistance, and Favors Microenvironment for Triple Negative Breast Cancer Tumor Metastasis
}

\section{Yi-Fang Tsai}

Comprehensive Breast Health Center, Department of Surgery, Taipei Veterans General Hospital

\section{Chi-Cheng Huang}

Comprehensive Breast Health Center, Department of Surgery, Taipei Veterans General Hospital

Yen-Shu Lin

Comprehensive Breast Health Center, Department of Surgery, Taipei Veterans General Hospital

Chih-Yi Hsu

Department of Pathology, Taipei Veterans General Hospital

\section{Ching-Po Huang}

Faculty of Medicine, School of Medicine, National Yang-Ming University

\section{Chun-Yu Liu}

Division of Medical Oncology, Department of Oncology, Taipei Veterans General Hospital

Jen-Hwey Chiu ( $\nabla$ chiujh@ym.edu.tw )

Comprehensive Breast Health Center https://orcid.org/0000-0003-3046-6250

\section{Ling-Ming Tseng}

Comprehensive Breast Health Center, Department of Surgery, Taipei Veterans General Hospital

\section{Research article}

Keywords: IL-17A, triple negative, breast cancer, anoikis, metastasis

Posted Date: August 14th, 2020

DOI: https://doi.org/10.21203/rs.3.rs-56413/v1

License: (1) (1) This work is licensed under a Creative Commons Attribution 4.0 International License. Read Full License 


\section{Abstract}

Background: Our previous study demonstrated that overexpression of multiple epidermal growth factorlike domains 11 was involved the recurrence mechanism of triple negative breast cancer (TNBC) via upregulation of cytokines and chemokines, including IL-17A signaling pathway. However, information concerning the role of IL-17A in tumor behavior or cancer microenvironment remains lacking. The aim of this study was to investigate the role of IL-17A on TNBC recurrent mechanisms, including tumor behavior, circulating tumor cells (CTCs), and cancer microenvironment.

Methods: Using human TNBC MDA-MB-231 and MDA-MB-468 lines, the role of IL17-A was elucidated by knocked down IL-17A (DIL-17A) or administration of different dose of IL-17A in the culture medium. Cell proliferation, migration assay, Western blot analysis and Real-time PCR for IL-17A related signaling were evaluated. Three groups of implanted 4T1 cells in BALB/c mice were designed, namely, wild type (WT), $D / L-17 A$, and $W T+$ neutralizing IL-17 antibody $(W T+A b)$. Tumor weight, necrosis area, and the number of CTCs were measured. Immunohistochemistry or Western blot for CD34, CD8, and TGF-b1 were evaluated. Anoikis resistance was analyzed by live/dead stain and flow cytometry. Finally, clinic-pathological correlation between IL-17A expression and patients' outcome such as disease free survival (DFS) and overall survival (OS) was performed by Kaplan-Meier's method.

Results: Our results demonstrated that IL-17A stimulated migratory activity, but not growth rate, of MDAMB-231/468 cells via increased Src, Rho, and COX2 expression. In vivo, there was an increased necrosis area, a decreased tumor CD34 expression and CTCs in DIL-17A group; while there was a decreased tumor CD34 expression, CD8(+) cells, and CTCs, but an increased TGF-b1 expression in WT+Ab group, compared to the WT group. Knocked down-IL-17A also decreased anoikis resistance in human TNBC and murine 4T1 cell lines. Kaplan-Meier's analysis disclosed a negative correlation between tumor IL-17A expression and OS in TNBC patients.

Conclusion: We conclude that IL-17A promotes migratory and angiogenic activity in the tumors, enhances anoikis resistance, and modulates the immune landscape of tumor microenvironment favoring subsequent cancer metastasis. The blockade of IL-17A might provide a co-treatment target to prevent tumor metastasis or recurrence in TNBC patients.

\section{Introduction}

Breast cancer is the most common diagnosed female cancer worldwide and ranks as the fourth most important cause of death in Taiwan [1]. Triple negative breast cancer (TNBC) represents $15 \%-20 \%$ of all newly diagnosed breast cancer patients and is characterized as a large tumor size, frequently diagnosed among younger women, and a poor prognosis with high metastases potential [2-4]. Recent investigations have demonstrated that TNBC may be composed of a number of distinct subtypes [5-7]. However, early recurrent mechanisms as consequences of poor prognosis in TNBC patients remain further elucidation. 
IL-17, a proinflammatory cytokine secreted by activated T cells, regulates the activities of MAPKs and NF$\mathrm{KB}$ and increases the expression of IL6 and cyclooxygenase-2 (COX-2) [8]. Overexpression of IL-17A is known to be highly associated with inflammatory diseases such as multiple sclerosis, psoriasis and rheumatoid arthritis [9]. It is generally accepted that chronic inflammation may increase the risk of cancer [10], as the inflammatory response shares various molecules and corresponding signaling pathways with the carcinogenic process. The IL-17 related protumoral effects on cancer initiation are postulated through MAPK and NF-kB recruitment. By contrast, IL-17 cytokines also act as an antitumor role and the same duality makes IL-17 a "double-edged sword" $[11,12]$. However, the role of IL-17A on the survival of circulating tumor cells (CTCs) is unclear.

Evidence suggests that poor prognosis in breast cancer might relate to CTCs [13,14], and the survival of tumor cells in circulation requires three aspects: 1.resistance to anoikis, 2. escape immune surveillance, and 3 . being stable under blood shearing force. The resistance to anoikis requires several signaling pathways related to anchorage- independent growth and epithelial-mesenchymal transition, including Akt, TrkB, Src, and so on [15]. Nonetheless, information concerning the role of IL-17A on the survival of CTCs remains to be elucidated.

Our previous results demonstrated that over-expression of MEGF11 in TNBC cells triggered the expression of many cytokines and chemokines, which effectively resulted in a cytokine cascade. Besides, a positive feedback between MEGF11 and IL-17A in TNBC cells was also demonstrated, which might explain the role of MEGF11 in TNBC recurrence [16]. Accordingly, the aim of this study was to investigate the role of IL-17A on TNBC recurrent mechanisms, including tumor behavior, cancer microenvironment, and the survival of CTCs.

\section{Methods}

\section{Cell line and reagents}

Human TNBC (ER-, HER2 low) cell lines MDA-MB-468 and MDA-MB-231 and the mouse mammary tumor 4T1 cell line [17], obtained from American Type Culture Collection (ATCC, Manassas, VA), were maintained in high glucose MEM, F12 MEM (NO.12400-024,Gibco, NY) and RPMI, respectively. They were supplemented with $10 \% \mathrm{FBS}, 2 \mathrm{mM}$ L-glutamine and penicillin/streptomycin and cultured at $37^{\circ} \mathrm{C}$ containing 5\% CO2. IL-17A (InvivoGen, San Diego, CA) and anti-mouse neutralizing IL-17A antibody (InVivoMab, Bio X Cell, BE0173, Lobanon, NH) were purchased commercially.

\section{Cell growth by trypan blue dye exclusion assay and cell cycle analysis}

MDA-MB-468/231 cells were cultured in low serum medium with a cell density $\left(1 \times 10^{5} /\right.$ well $)$ in a 12-well plate, followed by treatment of different doses of IL17A $(0-, 1-, 10 \mathrm{ng} / \mathrm{mL})$. After 1,2 , and 3 days of treatment, cells were washed with phosphate-buffered saline (PBS), $\mathrm{pH} 7.4$, and trypsinized with TE buffer (Gibco/Invitrogen, New York). Suspended cells were washed and cell numbers were counted with a 
hemocytometer. For cell cycle analysis, cells $\left(2 \times 10^{5} /\right.$ well) were cultured for $24 \mathrm{~h}$ in low serum medium ( $0.1 \% \mathrm{FBS})$, followed by another $24 \mathrm{~h}$-culture, and then cells were harvested for cell cycle analysis by flow cytometry. Cell cycles were presented as percentages of cell cycle fraction, namely, sub G0/G1 phase, G0/G1 phase, S phase, and G2/M phase.

\section{Cell migration assay}

In vitro cell migration of MDA-MB-468/231 cells was performed using a trans-well system (ThinCertTM cell culture inserts, 24 well, $8 \mu \mathrm{m}$, Greiner bio-one, Switzerland) [18]. In brief, MDA-MB-468/231 cells were cultured in the upper chamber and different doses of IL-17A $(0-, 1-, 10 \mathrm{ng} / \mathrm{mL})$ were administrated in the lower chamber. After $4 \mathrm{~h}$ (MDA-MB-231) or $8 \mathrm{~h}$ (MDA-MB-468) of culture, the cells on the reverse side of upper chamber membrane were fixed and stained with $2 \%$ crystal violet for $10 \mathrm{~min}$, then washed and photographed. The migrating cells were examined under a light microscope and quantified.

\section{Measurement of IL-17A level}

IL-17A level in the culture medium was quantified with ELISA method under the guidance of commercially available protocol (Quantikine ELISA Human IL-17A). In brief, MDA-MB-468/231 (4 × 10 $/$ well) cells were cultured for $1 \mathrm{~d}$, followed by administration of different doses $(0-, 1-, 10 \mathrm{ng} / \mathrm{mL})$ of IL-17A in low serum medium for $24 \mathrm{~h}$. Then, the culture medium were discarded and cells were cultured for another $48 \mathrm{~h}$, followed by harvesting the cells and the supernatants were collected, centrifuged ( $300 \times \mathrm{g})$ for $5 \mathrm{~min}$, and the final supernatants were ready for IL-17A measurement.

\section{Western blotting analysis}

Cultured cells were lysed in lysing buffer $(10 \mathrm{mM}$ Tris pH 7.4, $150 \mathrm{mM} \mathrm{KCl}, 150 \mathrm{mM} \mathrm{KCl}, 1 \%$ Triton X-100, phasphatase inhibitor and protease inhibitors cocktail (Complete Mini; Roche, Mannheim, Germany). Cell homogenates proteins were quantified using Bradford's method [19]. Thirty microgram of proteins were loaded to $10 \%$ SDS-PAGE and transferred to a PVDF membrane, which was blocked with $5 \%$ skimmed milk and probed with specific primary antibodies such as IL-17A (\#3171, R\&D Systems, Inc. Minneapolis, MN), Src (\#60521, GeneTex, Inc., Irvine, CA), phosphor-Src (\#6943, Cell Signaling Technology, Beverly, MA), COX-2 (\#12282, Cell Signaling), phospho-AKT (\#9271, Ser473, Cell Signaling), AKT (\#9272, Cell Signaling), cdc42 (\#2466, Cell Signaling), RhoA (\#2117, Cell Signaling), RhoC (\#3430, Cell Signaling), and anti- $\beta$-actin (\#3700S, Cell Signaling). The blots were then washed, soaked with anti-Rabbit IgG HRP-linked secondary antibodies or anti-Mouse IgG HRP-linked secondary antibodies (Cell Signaling Technology, Beverly, MA, USA.) After $1 \mathrm{~h}$ hybridization, membranes were washed and developed with an ECL detection kit (Amersham Pharmacia Biotech Inc., NJ) and quantified by Multi-Gauge software analysis (Fuji Photo Film Co, Ltd, Tokyo, Japan). The $\beta$-actin was used as internal control in all experiments.

\section{RNA extraction and reverse transcription-PCR}

Total RNA were isolated by using a modified single-step guanidinium thiocyanate method [20]. (TRI REAGENT, T-9424, Sigma Chem. Co., St. Louis, MO) Complementary DNA (cDNA) was prepared from the total RNA complied with the First Strand cDNA Synthesis Kit (Invitrogen, CA). The de novo gene synthesis 
changed by each treatment group was detected by reverse transcriptase-polymerase chain reaction (RTPCR). Primers pairs were purchased commercially[16]. The possible contamination of any PCR component was excluded by performing a PCR reaction in the absence of RT product in each set of experiment. Quantification of RNA transcripts was analyzed according to the method described previously [16]. For statistical comparison, the relative expression of specific genes mRNA was normalized to the amount of GAPD in the same RNA extracts. All samples were analyzed in triplication.

\section{Short hairpin RNA (shRNA) transfection}

Short hairpin RNA (shRNA) used to silence IL-17A genes was obtained from Academia Sinica. One day after MDA-MB-468/231 lines and murine 4T1 cells were subcultured, they (30-40\% confluent) were transfected for $24 \mathrm{~h}$ with shRNA against IL-17A gene or non-silencing control using GenePORTER 2 transfection reagent (Genlantis, San Diego, CA) dissolved in Optimem (Invitrogen, CA) at a final concentration of $80 \mathrm{nM}$. And then, MDA-MB-468/231 and 4T1 cells were recovered for further experiments. After several passages, $\Delta / L-17 A$ cell lines were established by puromycin selection. The transfection efficiency was validated by Western blot analysis.

\section{Measurement of anoikis resistance}

Anoikis resistance was evaluated using adherent plate and non-adherent (anoikis) plate commercially (CBA081m Cell Biolabs, San Diego, CA). Briefly, MDA-MB-231, MDA-MB-468, and murine 4T1 cells at a density of $2 \times 10^{4} /$ well were cultured in adherent- and anoikis plates, respectively. After cells were cultured for $16 \mathrm{~h}$ (4T1 cells), $24 \mathrm{~h}, 48 \mathrm{~h}$ (MDA-MB-231), mixed live (Calcein -AM) and dead (Ethidium homodimer-1) staining solution (L3224, Invitrogen Detection Technologies, Eugene, OR) was added into the cultured medium for 30-45 min, followed by quantification of dead cells with a fluorescent microscopy. For flow cytometry analysis (MDA-MB-468), the protocols were the same as above-mentioned ones except the cell density being $2 \times 10^{5} /$ well.

\section{In vivo tumor xenograft}

In this study, any protocols that involved experimental mice followed the ARRIVE (Animal Research: Reporting of In Vivo Experiments) guidelines and were approved by the Institutional Animal Committee of Taipei Veterans General Hospital (No. 2018-029). Immunodeficient NU-Foxn1nu 8-wk-old mice were obtained from the National Laboratory Animal Center (Taipei, Taiwan, ROC). They were given ad libitum access to water and food and maintained in a specific pathogen-free environment with a $12 \mathrm{~h}$ dark-light cycle at $22-24^{\circ} \mathrm{C}$ with $50 \%$ humidity. Wild type (WT) and knocked down IL-17A ( $\left.\Delta / L-17 A\right)$ MDA-MB-231 cells $\left(1 \times 10^{7}\right.$ in $0.1 \mathrm{~mL}$ PBS$)$ containing a luciferase gene were injected into back of immunodeficient NUFoxn1nu mice, which gave rise to a solid tumour that was noticeable around the injection site. At the 2nd, $3 \mathrm{rd}$, and 5th week, the progression of the tumor was visualized using an in vivo imaging system (IVIS).

\section{In vivo metastasis study}

Three groups of animal studies were designed, namely, WT, knocked down IL-17A ( $\Delta / L-17 A)$, and anti-IL17A neutralizing antibody $(250 \mu \mathrm{g} / 100 \mu \mathrm{L}$, twice a week) (WT + anti-IL-17A antibody). Mouse mammary 
4T1 cells $\left(5 \times 10^{6}\right.$ in $0.1 \mathrm{~mL}$ PBS) were orthotopically injected into the fat pads (left upper and/or right lower mammary glands) of 8-wk-old female BALB/c mice. The mice were sacrificed at 8 weeks thereafter or when the tumour sizes being more than $2 \%$ of the body weight. The tumor sizes and weights were measured, and the circulating tumor cells were isolated as mentioned below.

\section{Selection of circulating mammary breast cancer $4 \mathrm{~T} 1$ cells}

After the anaesthetized 4T1 bearing mice were sacrificed, blood cells were obtained and centrifuged (400 g) in Ficoll-Paque PREMIUM (density: 1.084) (17-5446-02, GE Healthcare Bio-Sciences, Sweden) gradient medium. The peripheral mononuclear cells were subjected to primary culture. After several passages, circulating 4T1 cells were selected using 6-thioguanine $(60 \mu \mathrm{M})(\mathrm{A} 4882$, Sigma-Aldrich, MO, USA) [21], which was followed by quantification with a 2-hydroxyethyl agarose colony assay (A4018, Sigma-Aldrich, MO, USA). A colony was defined as a blue dye-stained group of cells that was $\geq 1 \mathrm{~mm}$.

\section{Subjects}

Under the approval of the Institutional Review Board of Taipei Veterans General Hospital (2013-10020BC), human tumour tissues from a bio-bank were obtained. From Jan. 2001 to Dec. 2010, eighty three patients diagnosed as female breast cancer in department of pathology, Taipei Veterans General Hospital were enrolled. Records such as oestrogen receptor (ER) status, progestin receptor (PR) status, HER2 status and clinical outcome, including overall survival (OS) and disease-free survival (DFS), were retrospectively reviewed. All data had been collected during clinical care and did not involve direct contact with the patients. Besides, written consent by the study subjects was waived by the Institutional Review Board. Every subject participated in this study $(n=83)$ was followed up for $\geq 5$ years. Patients' outcome was subjected to a Kaplan-Meier survival analysis. ER or PR values of $\geq 1 \%$ were defined as positive, while ER or PR values of $<1 \%$ were defined as negative.

\section{Immunohistochemical analysis}

The protein expression levels of IL-17A from a tissue array (83 tumour samples) were assessed by immunohistochemical staining for IL-17A (Genetex, GTX49102). The results were analysed by one pathologist over a short period of time (2 months). The protein expression of IL-17A was semi-quantified and expressed as $(0),<10 \%,(1), 11-25 \%,(2), 26-50 \%$, and (3) $>50 \%$ of the tumour cells examined.

On the animal tumour tissues, the protein expression levels of CD34, CD8, and TGF- $\beta 1$ were assessed by immunohistochemical staining for CD34 (\#65867, Genetex, Inc., Irvine, CA) and CD8 (\#98941, Cell Signaling, Danvers, MA), and TGF- $\beta 1$ (\#130023, Genetex Inc., Irvine, CA) respectively. The CD8 (+) cells were quantified by counting the cells within an area of $1 \mathrm{~mm}^{2}$, while the CD34 and TGF- $\beta 1$ were quantified by Western blot analysis.

\section{Statistic analysis}

Results were expressed as the mean \pm SEM. Differences between two groups were identified by MannWhitney $U$ test or Student $t$ test. Differences between groups on each time point or each dose were 
identified by one-way ANOVA, followed by Dunnet's post hoc test. Statistical comparison between two independent variables was determined by Two-way ANOVA with Bonnforonni post-hoc to correct multiple comparisons. A $p$-value $<0.05$ is considered statistically significant compared to vehicle or no treatment group.

DFS was defined as the time between initial breast cancer diagnosis and the date of recurrence as confirmed by pathology or an imaging study. OS was calculated from the time of initial breast cancer diagnosis to the date of death or last visit in outpatient clinic. The Kaplan-Meier method was used to estimate the cumulative incidence of RFS and OS, and log-rank tests were then used for the various comparisons (GraphPad Prism 5).

\section{Results}

\section{Role of IL-17A in tumor behavior}

Knocked-down IL-17A did not affect cell growth in human TNBC cell lines.

After IL-17A gene was silenced in TNBC MDA-MB-231 and MDA-MB-468 lines, there was no significant change of growth rate in $\triangle I L-17 A$ group compared to the WT group, both in cell lines studies (Fig. 1a) and in tumor-bearing nude mice studies (Fig. 1b).

\section{IL-17A did not affect cell growth but promoted cell migration in TNBC lines}

When IL-17A (0-, 1-, $10 \mathrm{ng} / \mathrm{mL})$ was administrated in the culture medium of MDA-MB-231 and MDA-MB468, there was an increased cell number in 1- or 2-, but not 3-d culture (Fig. 2a, 2-way ANOVA). Cell cycle analysis demonstrated no significant change of cell cycle fractions such as G0G1- or S + G2M phase (Fig. 2b, one way ANOVA).

By Western blot analysis (Fig. 2c), the results showed that IL-17A dose-dependently increased p-Src, but not AKT, ERK, CDK4, and cyclin D1 protein expression in both TNBC lines (Fig. 2d) .By contrast, IL-17A (0-, $1-, 10 \mathrm{ng} / \mathrm{mL}$ ) dose-dependently increased migratory activity (Fig. 3a) via activation of Rho A, CDC42, COX2, but not Rho $C$, in MDA-MB-231, while activation of Rho A, COX2, but not Rho $C$ and CDC42, in MDAMB-468 line (Fig. 3b).

\section{IL-17A up-regulated gene expression of pro-inflammatory cytokines and chemokines}

When IL-17A was administrated in the culture medium of TNBC cells, there was an increased mRNA transcripts of pro-inflammatory cytokines (TNF-a, IL-1 $\beta$ ) and chemokines (CXCL2, CCL20) and COX2 in MDA-MB-231 and MDA-MB-468 (Fig. 3c). Of note, IL-17A up-regulated IL-17A gene expression both at 
transcript level and protein level (Fig. 3d). These data suggest that IL-17A has autoregulation activity and enhances inflammatory cytokines and chemokines cascades.

\section{In vivo metastasis study}

\section{Role of IL-17A on the tumor behaviour}

Using murine mammary 4T1 cell line as in vivo metastatic model, three groups of animal studies were designed, namely, WT, knocked down IL-17A $(\Delta / L-17 A)$, and WT + anti-IL-17A neutralizing antibody (WT + $A b)$. The roles of IL-17A in tumors ( $\triangle / L-17 A$ group) and around tumor microenvironment (WT + Ab group) were compared to the WT group. The results showed that there was a significant decreased tumor volume and an increased tumor necrosis area (Fig. 4a) in $\Delta / L-17 A$ group compared to the WT group. To investigate the role of IL-17A on angiogenesis in vivo, CD34 expression by immunohistochemistry and Western blot was performed. The results demonstrated that there was a significant decreased CD34 expression (Fig. 4b) in $\Delta / L-17 A$ and $W T+A b$ groups compared to the WT group.

\section{Role of IL-17A on tumor microenvironment}

When dissecting the tumors away from the mice chest wall, it was surprising to notice that the tumors of the WT + Ab group, which were no smaller in size than those of the WT group, were easily freed from adjacent chest wall without any adhesion nor increased vascularity (Fig. 4a). To investigate the role of IL17A on immune landscape in vivo, CD 8 and TGF- $\beta 1$ expressions by immunohistochemistry and TGF- $\beta 1$ expression by Western blot were performed. The results demonstrated that there was a significant decreased CD8 (+) T cells (Fig. 5a), and also decreased COX2 but increased TGF- $\beta 1$ expression in $\Delta / L-$ $17 A$ and $W T+A b$ groups compared to the WT group (Fig. $5 b)$.

Role of IL-17A on anoikis resistance in circulating tumor cells (CTCs)

The CTCs obtained from the blood of sacrificed mice were isolated, subcultured, and selected by colony assay. The results disclosed that there were significant decreased CTCs colonies in both $\triangle I L-17 A$ group and WT + Ab group compared to the WT group (Fig. 6a). Using anoikis plate as in vitro model, the results showed that there was a decreased anoikis resistance via caspase 3 activation (Fig. 6b) in $\Delta / L-17 A 4 T 1$ group compared to the WT 4T1 group. Similarly, there was a decreased anoikis resistance (Fig. 6c) in $\Delta / L$ 17A MDA-MB-231/468 group compared to the WT MDA-MB-231/468 group. These data suggest that IL17A plays important roles in maintaining CTCs survival against anoikis. Clinically, the expression of IL17A correlated negatively with patients' OS (Fig. 7a), but a trend with patients' DFS (Fig. 7b).

\section{Discussion:}

Using paired (non-recurrent and recurrent) resected tumor tissues obtained from breast cancer patients, we have identified a novel gene, MEGF11, is highly associated with tumor recurrence in TNBC patients. Besides, Overexpression of MEGF11 gene up-regulates IL-17A gene expression in TNBC MDA-MB-231 and 
MDA-MB-468 cells. Although IL-17A is well known to be highly associated with inflammatory diseases such as rheumatoid arthritis and psoriasis [9], the role of IL-17A in TNBC recurrence remains further elucidation. In this study, we have demonstrated that IL-17A promotes migration-promoting activity in the tumors and changes the immune landscape favoring tumor microenvironment for cancer metastasis.

While knocked down IL-17A group was designed to investigate the role of IL-17A in tumor behavior, the WT + anti-IL17A antibody group was designed to elucidate that in tumor microenvironment. Tumor necrosis, a form of cell death, is commonly associated with hypoxia, rapidly growing, and aggressive forms of breast cancer [22]. There is evidence that angiogenesis is essential for tumor growth and metastasis [23]. and IL-17A promotes tumor angiogenesis via stimulation of endothelial fatty acid $\beta$ oxidation [24]. Using CD34 as a marker for hematopoietic stem cells [25], our results showing a significantly increased necrosis area and a decreased CD34 expression in $\triangle I L-17 A$ group compared to the WT group suggest that IL-17A enhances angiogenesis and hence against ischemic necrosis in rapid progress tumor tissue.

By contrast, when dissecting the tumors away from the mice chest wall, tumors of the WT + Ab group were easily detached from adjacent chest wall without any adhesion nor increased vascularity (Fig. 5A). A decreased CD34 expression level supports the speculation of decreased angiogenesis in this group. Recent investigations suggest that the immune system plays a dual role in tumor progression, namely, inhibiting or promoting tumor expansion. For example, TGF- $\beta 1$ produced during chronic inflammation actively promotes growth and metastasis $[10,26]$. Besides, the TGF- $\beta$ signaling pathway plays key roles in the early development of Treg cells in the thymus [27], in maintaining the homeostasis of peripheral CD4 + T cells via by increasing IL-7Ra expression [28], and in controlling CD8 ${ }^{+}$T-cell homeostasis [29]. Of note, several lines of evidence demonstrate the opposing effects of TGF- $\beta$ on Th17 cells, namely, the immunoregulatory or pathogenic role of the Th17 cells in clinical settings [30,31].

Our results that there was a decreased CD8(+) cells and an increased TGF- $\beta$ expression in WT + Ab (antiIL-17A) group lead us to speculate that TGF- $\beta$ plays an immunosuppressive role leading to immune escape of the implanted 4T1 tumors, which is in agreement with previous investigation [32].

The presence of circulating tumor cells (CTCs) is well known as a mechanism of tumor metastasis or cancer recurrence [33]. Several hypotheses about CTCs evading immune surveillance have been proposed, such as adhered to platelet and myeloid-derived suppressor cells [34, 35], or mediated by PD-L1 [36]. Our results that there was significantly decreased CTCs colony number in $\triangle / L-17 A$ and WT + antiIL17A Ab groups compared to the WT group demonstrated a negative correlation of IL-17A expression and CTCs in the blood, which is consistent with previous findings observed in colon cancer [37].

There is consensus that persistent chronic inflammation increases the risk of cancer [10] and necrosis has proinlfammatory and tumor promoting potential [38]. The use of nonsteroidal anti-inflammatory drugs (NSAIDs) is well demonstrated to decrease incidence and mortality of many cancers [39]. IL-17E/IL17E receptor axis may underlie TNBC resistance to EGFR inhibitors and suggest that inhibiting IL-17E or 
its receptor in combination with EGFR inhibitor administration may improve TNBC management [40]. Recent studies suggest that IL-17A inhibitor might play important role in immune therapies for psoriasis and ankylosing spondylitis patients [41].

\section{Conclusion}

Our results demonstrate that IL-17A stimulates migratory activity of TNBC cells and modulates the immune landscape of tumor microenvironment favoring subsequent cancer metastasis. The blockade of IL-17A might provide a co-treatment target to prevent tumor metastasis or recurrence in TNBC patients.

\section{Declarations}

Ethics approval and consent to participate: Study protocols involving experimental mice followed ARRIVE (Animal Research: Reporting of In Vivo Experiments) guidelines and were approved by the Institutional Animal Committee of and Taipei Veterans General Hospital (No. 2018-029).

The human study for tumour tissue utilization from the bio-bank was approved by the Institutional Review Board of Taipei Veterans General Hospital (\# 2013-10-020BC).

Consent of publication: Not applicable

Data availability: All data generated or analysed during this study are included in this published article (and its Supplementary Information files)

Competing interest: We declare that we have no conflicts of interest, including financial and non-financial interests such as the following items.

- Unpaid membership in a government or non-governmental organization

- Unpaid membership in an advocacy or lobbying organization

- Unpaid advisory position in a commercial organization

- Writing or consulting for an educational company

- Acting as an expert witness

\section{Author contributions:}

Chiu, JH formed the idea. Tseng, LM and Chiu, JH contributed equally in this manuscript. Tseng, LM supervised the experiments. Liu, CY, Tseng, LM, Tsai, YF, Huang CC and Lin YS provided clinical samples and data. Huang, CP performed the experiments. Hsu, CY read the pathology slices.

Funding: This work was supported by grants from the Ministry of Health and Welfare (Center of Excellence for Cancer Research at Taipei Veterans General Hospital phase II, MOHW108-TDU-B-212112015, phase III, MOHW109-TDU-B-212-010001). 
Acknowledgements: We are in debt to Chou, SS, Wang, YL, and Hsu, TH for their technique supports. This work was supported by the biobank from the Division of Experimental Surgery, Department of Surgery, Taipei Veterans General Hospital. We thank the Taiwan Animal Consortium (MOST 107-2319-B-001-002)-Taiwan Mouse Clinic, which is funded by the Ministry of Science and Technology (MOST) of Taiwan, for technical support in the IVIS animal experiment.

\section{References}

1. Nik-Zainal S, Davies H, Staaf J, et al. Landscape of somatic mutations in 560 breast cancer wholegenome sequences [published correction appears in Nature. 2019 Feb;566(7742):E1]. Nature. 2016;534(7605):47-54. doi:10.1038/nature17676.

2. Podo, F., et al., Triple-negative breast cancer: Present challenges and new perspectives. Molecular Oncology, 2010. 4(3): p. 209-229.

3. Sharma, P., Biology and Management of Patients With Triple-Negative Breast Cancer. The oncologist, 2016. 21(9): p. 1050-1062.

4. Thike, A.A., et al., Triple-negative breast cancer: clinicopathological characteristics and relationship with basal-like breast cancer. Modern Pathology, 2010. 23(1): p. 123-133.

5. Lehmann, B.D., J.A. Pietenpol, and A.R. Tan, Triple-Negative Breast Cancer: Molecular Subtypes and New Targets for Therapy. American Society of Clinical Oncology Educational Book, 2015(35): p. e31-e39.

6. Abramson, V.G., et al., Subtyping of triple-negative breast cancer: Implications for therapy. Cancer, 2015. 121(1): p. 8-16.

7. Tseng, L.-M., et al., A comparison of the molecular subtypes of triple-negative breast cancer among non-Asian and Taiwanese women. Breast Cancer Research and Treatment, 2017. 163(2): p. 241-254.

8. <Herpesvirus Saimiri encodes a new cytokine, IL-17, which binds to a novel cytokine receptor..pdf>.

9. Jung, M.K., J.-E. Kwak, and E.-C. Shin, IL-17A-Producing Foxp3 + Regulatory T Cells and Human Diseases. Immune Netw, 2017. 17(5): p. 276-286.

10. Landskron, G., et al., Chronic inflammation and cytokines in the tumor microenvironment. J Immunol Res, 2014. 2014: p. 149185.

11. Fabre, J.A.S., et al., The Interleukin-17 Family of Cytokines in Breast Cancer. Int J Mol Sci, 2018. 19(12).

12. Welte, T. and X.H. Zhang, Interleukin-17 Could Promote Breast Cancer Progression at Several Stages of the Disease. Mediators Inflamm, 2015. 2015: p. 804347. 
13. <Circulating tumor cells, disease progression, and survival in metastatic breast cancer..pdf>.

14. Zhang, Y., et al., Role of Circulating Tumor Cell (CTC) Monitoring in Evaluating Prognosis of TripleNegative Breast Cancer Patients in China. Medical science monitor : international medical journal of experimental and clinical research, 2017. 23: p. 3071-3079.

15. Paoli, P., E. Giannoni, and P. Chiarugi, Anoikis molecular pathways and its role in cancer progression. Biochimica et Biophysica Acta (BBA) - Molecular Cell Research, 2013. 1833(12): p. 3481-3498.

16. Chiu, J.-H., et al., MEGF11 is related to tumour recurrence in triple negative breast cancer via chemokine upregulation. Scientific Reports, 2020. 10(1): p. 8060.

17. Pulaski, B.A. and S. Ostrand-Rosenberg, Mouse 4T1 Breast Tumor Model. Current Protocols in Immunology, 2000. 39(1): p. 20.2.1-20.2.16.

18. Chiu, J.H., et al., Effects of Chinese medicinal herbs on expression of brain-derived Neurotrophic factor (BDNF) and its interaction with human breast cancer MDA-MB-231 cells and endothelial HUVECs. BMC Complement Altern Med, 2017. 17(1): p. 401.

19. Bradford, M.M., A rapid and sensitive method for the quantitation of microgram quantities of protein utilizing the principle of protein-dye binding. Analytical Biochemistry, 1976. 72(1): p. 248-254.

20. Chomczynski, P. and N. Sacchi, The single-step method of RNA isolation by acid guanidinium thiocyanate-phenol-chloroform extraction: twenty-something years on. Nature Protocols, 2006. 1(2): p. 581-585.

21. Huang, S. and P.J. Houghton, Targeting mTOR signaling for cancer therapy. Current Opinion in Pharmacology, 2003. 3(4): p. 371-377.

22. Tata, A., et al., Rapid Detection of Necrosis in Breast Cancer with Desorption Electrospray Ionization Mass Spectrometry. Scientific Reports, 2016. 6(1): p. 35374.

23. Folkman, J., Tumor Angiogenesis: Therapeutic Implications. New England Journal of Medicine, 1971. 285(21): p. 1182-1186.

24. Wang, R., et al., IL-17A-stimulated endothelial fatty acid $\beta$-oxidation promotes tumor angiogenesis. Life Sciences, 2019. 229: p. 46-56.

25. Sidney, L.E., et al., Concise Review: Evidence for CD34 as a Common Marker for Diverse Progenitors. STEM CELLS, 2014. 32(6): p. 1380-1389.

26. <The hallmarks of successful anticancer immunotherapy.pdf>.

27. Ouyang, W., et al., Transforming Growth Factor- $\beta$ Signaling Curbs Thymic Negative Selection Promoting Regulatory T Cell Development. Immunity, 2010. 32(5): p. 642-653. 
28. Ouyang, W., et al., TGF- $\beta$ Cytokine Signaling Promotes CD8 + T Cell Development and Low-Affinity CD4 + T Cell Homeostasis by Regulation of Interleukin-7 Receptor a Expression. Immunity, 2013. 39(2): p. 335-346.

29. <Transforming growth factor-beta pathway serves as a primary tumor suppressor in CD8 $+T$ cell tumorigenesis..pdf>.

30. Gagliani, N., et al., Th17 cells transdifferentiate into regulatory T cells during resolution of inflammation. Nature, 2015. 523(7559): p. 221-225.

31. Sharma, M., S.V. Kaveri, and J. Bayry, Th17 cells, pathogenic or not? TGF- $\beta 3$ imposes the embargo. Cellular \& Molecular Immunology, 2013. 10(2): p. 101-102.

32. Nam, J.S., et al., Transforming growth factor beta subverts the immune system into directly promoting tumor growth through interleukin-17. Cancer Res, 2008. 68(10): p. 3915-23.

33. Gupta, G.P. and J. Massagué, Cancer Metastasis: Building a Framework. Cell, 2006. 127(4): p. 679695.

34. Lou, X.-L., et al., Interaction between circulating cancer cells and platelets: clinical implication. Chinese journal of cancer research = Chung-kuo yen cheng yen chiu, 2015. 27(5): p. 450-460.

35. Liu, Q., Q. Liao, and Y. Zhao, Myeloid-derived suppressor cells (MDSC) facilitate distant metastasis of malignancies by shielding circulating tumor cells (CTC) from immune surveillance. Medical Hypotheses, 2016. 87: p. 34-39.

36. Mazel, M., et al., Frequent expression of PD-L1 on circulating breast cancer cells. Molecular Oncology, 2015. 9(9): p. 1773-1782.

37. Tseng, J.Y., et al., Interleukin-17A modulates circulating tumor cells in tumor draining vein of colorectal cancers and affects metastases. Clin Cancer Res, 2014. 20(11): p. 2885-97.

38. Hanahan, D. and Robert A. Weinberg, Hallmarks of Cancer: The Next Generation. Cell, 2011. 144(5): p. 646-674.

39. Yan, L., et al., Therapeutic potential of cytokine and chemokine antagonists in cancer therapy. European Journal of Cancer, 2006. 42(6): p. 793-802.

40. Merrouche, Y., et al., IL-17E synergizes with EGF and confers in vitro resistance to EGFR-targeted therapies in TNBC cells. Oncotarget, 2016. 7(33): p. 53350-53361.

41. Reis, J., R. Vender, and T. Torres, Bimekizumab: The First Dual Inhibitor of Interleukin (IL)-17A and IL$17 F$ for the Treatment of Psoriatic Disease and Ankylosing Spondylitis. BioDrugs, 2019. 33(4): p. $391-$ 399. 

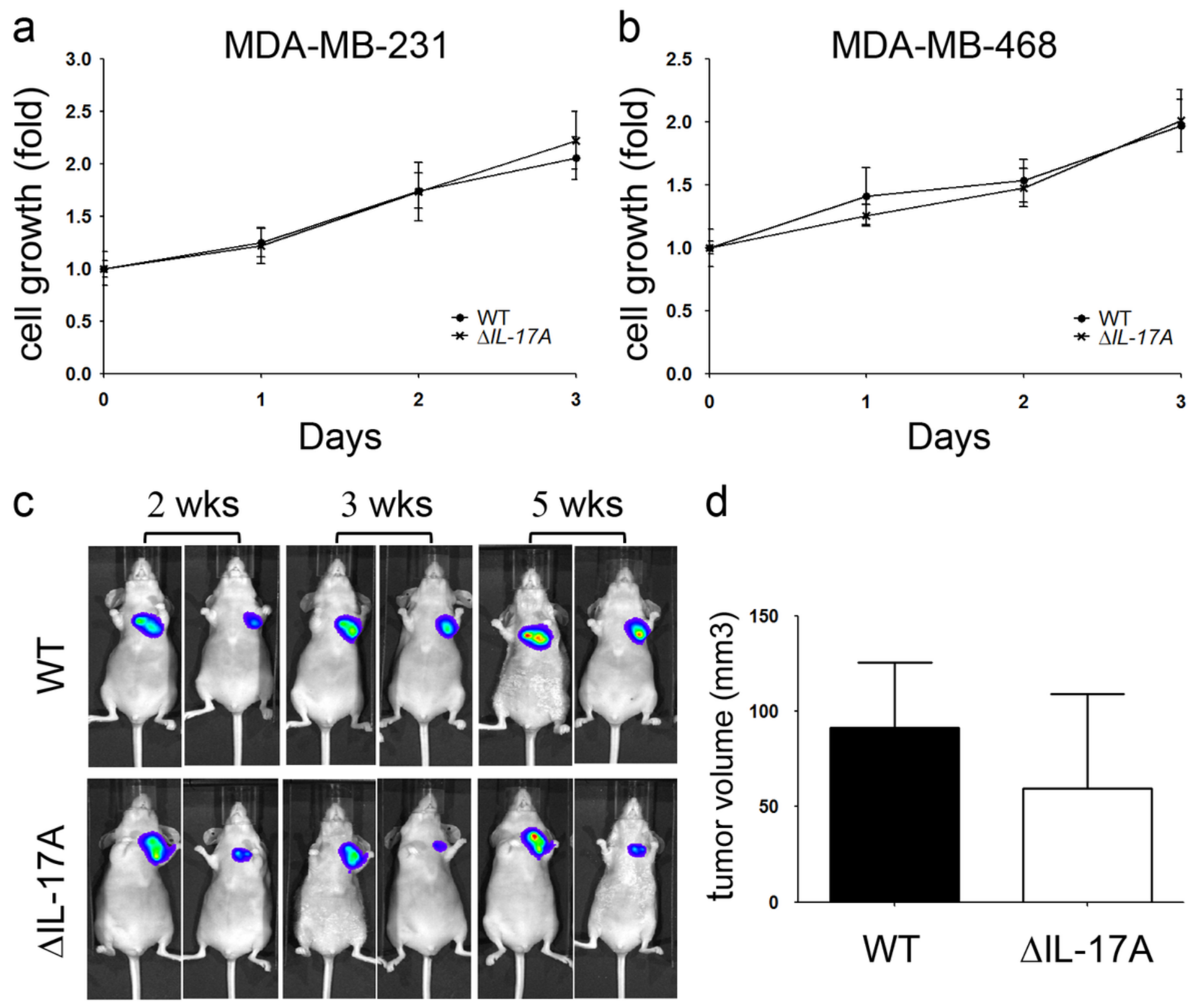

Figure 1

The role of IL-17A on cell growth in MDA-MB-231 and MDA-MB-468 cell lines. After IL-17A was knocked down in MDA-MB-231 and MDA-MB-468 cells (a), cell proliferation was evaluated by trypan blue exclusion assay. Wild type (WT) and knocked down IL-17A ( $\Delta$ IL-17A) MDA-MB-231 cells (1x107 in $0.1 \mathrm{~mL}$ PBS) containing a luciferase gene were injected into back of immunodeficient NU-Foxn1nu mice. At the 2nd, 3rd, and 5th week, the progression of the tumors was visualized using an in vivo imaging system (IVIS) and tumour volume was quantified (b). Two-way ANOVA and Mann-Whitney $U$ test were used for statistical analysis for cell growth in vitro and in vivo, respectively. 

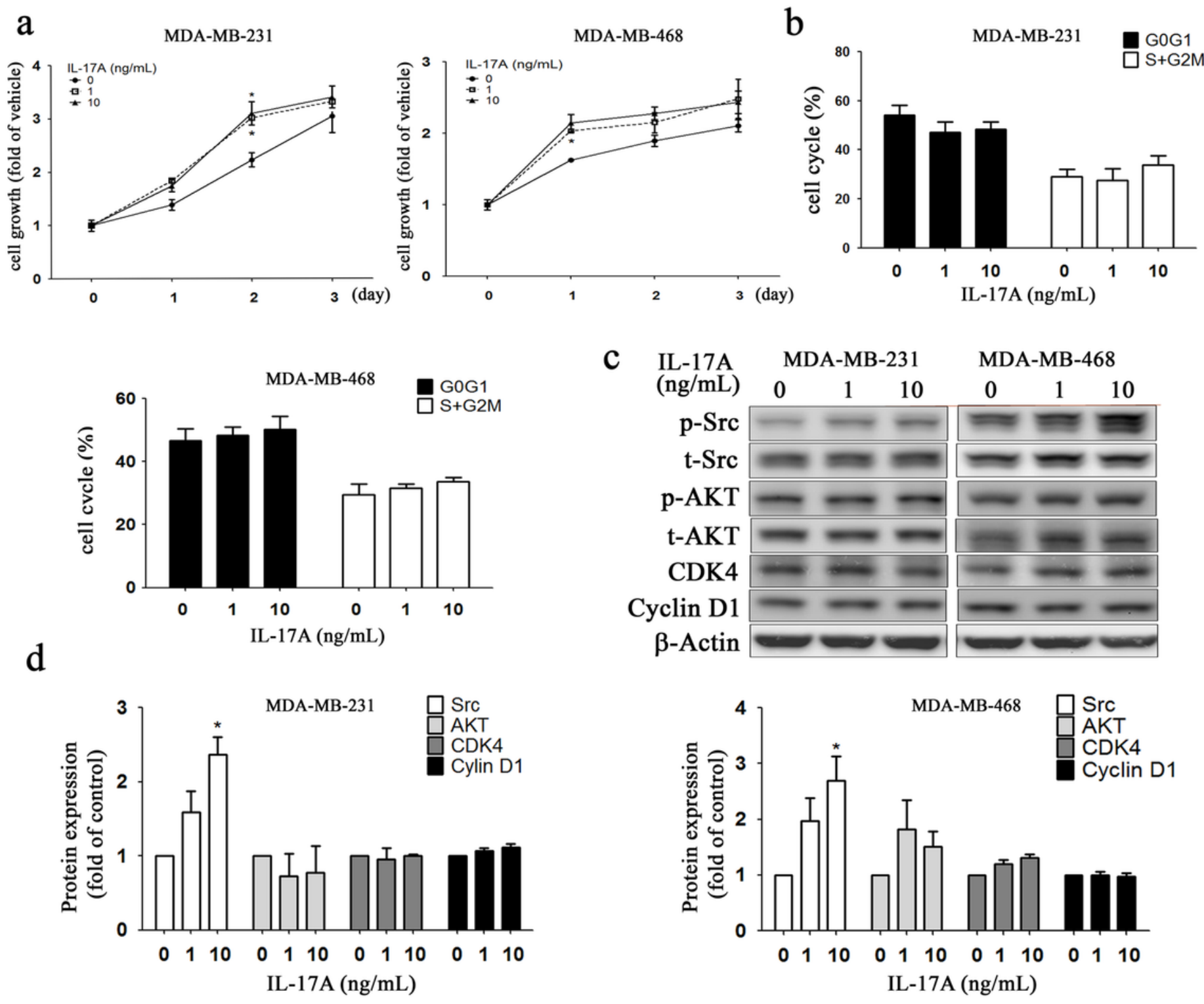

Figure 2

Effects of exogenously administration of IL-17A on cell growth in MDA-MB-231 and MDA-MB-468 cell lines. MDA-MB-468/231 cells were cultured in low serum medium with a cell density ( 1 x 104 / well), followed by treatment of different doses of IL17A (0-, 1-, $10 \mathrm{ng} / \mathrm{mL})$. After 1, 2, and 3 days of treatment, cell proliferation rate was evaluated by trypan blue assay (a). For cell cycle analysis, cells ( $2 \times 105$ / well) were cultured for $24 \mathrm{~h}$ in low serum medium, followed by another $24 \mathrm{~h}$-culture, and then cells were harvested for cell cycle analysis (b). Cell cycles were presented as percentages of cell cycle fraction, namely, sub G0/G1 phase, G0/G1 phase, S phase, and G2/M phase. Growth related signalling proteins expression, such as Src, AKT, CDK4, and cyclinD1, were analyzed by Western blot (c) and quantified (d). Asterisk indicates a $p$ value $<0.05$ (one-way ANOVA). 
a

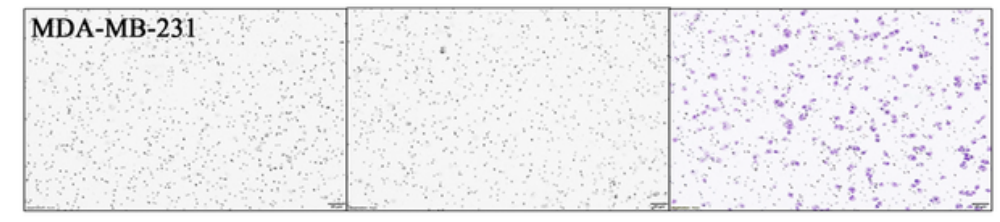

IL-17A 0 ng/mL

IL-17A $1 \mathrm{ng} / \mathrm{mL}$

IL-17A $10 \mathrm{ng} / \mathrm{mL}$
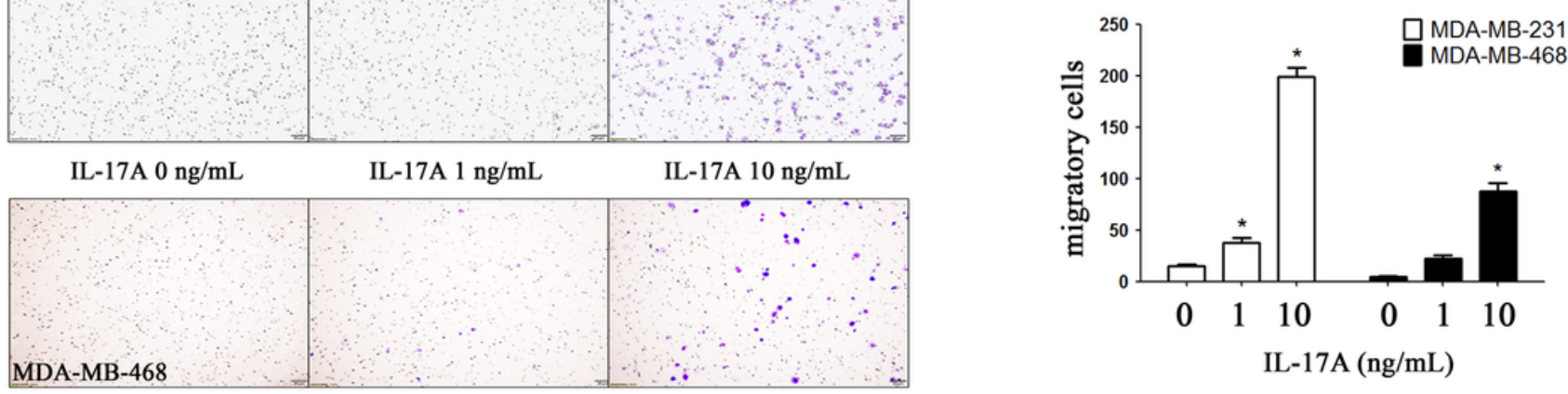

b

\begin{tabular}{cccccccc} 
IL-17A & \multicolumn{2}{c}{ MDA-MB-231 } & \multicolumn{2}{c}{ MDA-MB-468 } \\
(ng/mL) & 0 & 1 & 10 & 0 & 1 & 10 \\
\hline
\end{tabular}

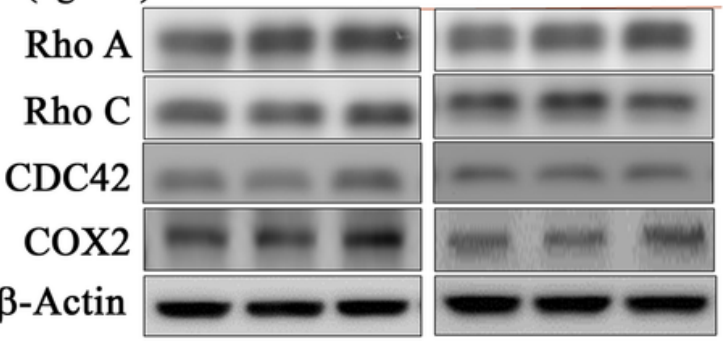

C

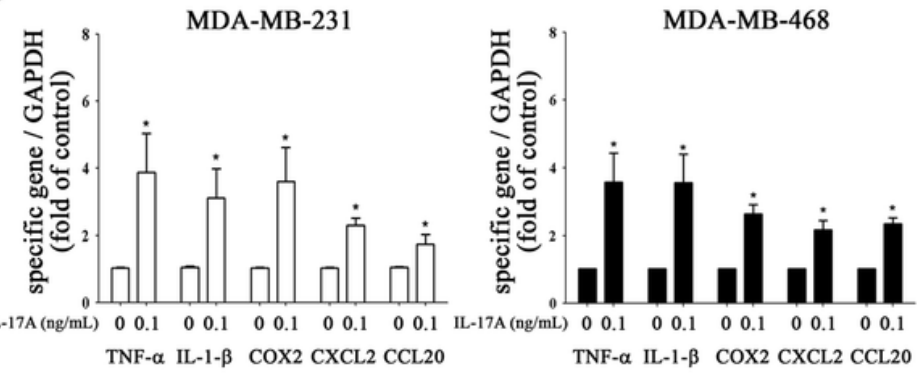

MDA-MB-231

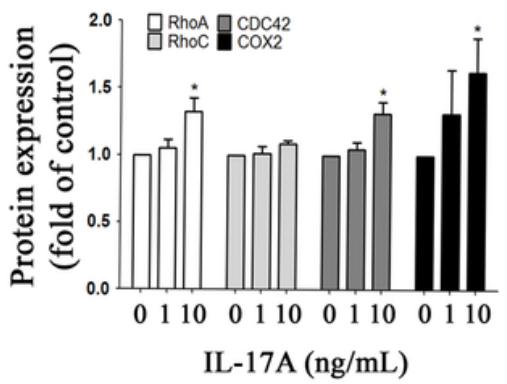

d
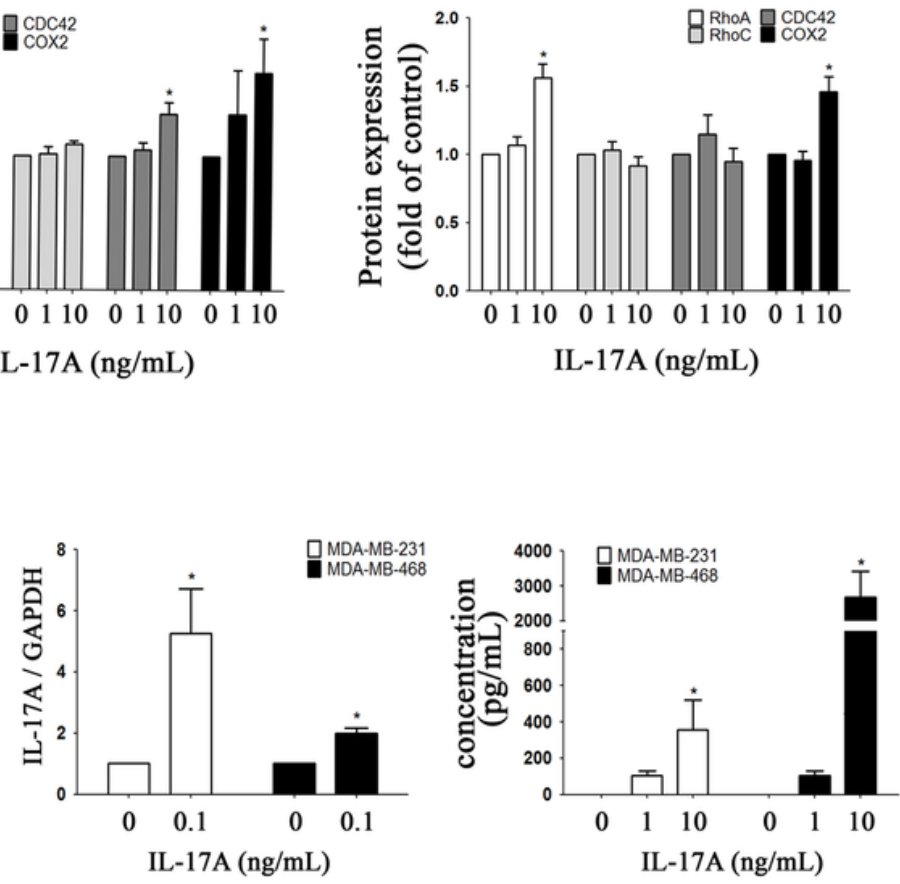

Figure 3

Effects of exogenously administration of IL-17A on cell migration, gene expression of proinflammatory cytokines and chemokines in MDA-MB-231 and MDA-MB-468 cell lines. MDA-MB-468/231 cells were cultured in a trans-well system as described in Method, followed by administration of different doses of IL17A (0-, 1-, $10 \mathrm{ng} / \mathrm{mL})$ in the lower chamber. After 4-8 $\mathrm{h}$, the migratory cells were photographed, quantified (one-way ANOVA) (a). For Western blot analysis, cells $(1 \times 106 /$ well) were cultured for $24 \mathrm{~h}$ in low serum medium, followed by another $24 \mathrm{~h}$-culture, and then cells were harvested and probed with specific antibodies, quantified (one-way ANOVA) (b). mRNA transcripts of pro-inflammatory cytokines (TNF- $a, I L-1 \beta$ ) and chemokines (CXCL2, CCL20) and COX2 were analyzed with real-time PCR (MannWhitney $U$ test) (c). IL-17A gene expression including mRNA and protein level (d) were quantified by realtime PCR and ELISA assay (one-way ANOVA), respectively. Asterisk indicates a $p$ value $<0.05$. 
a
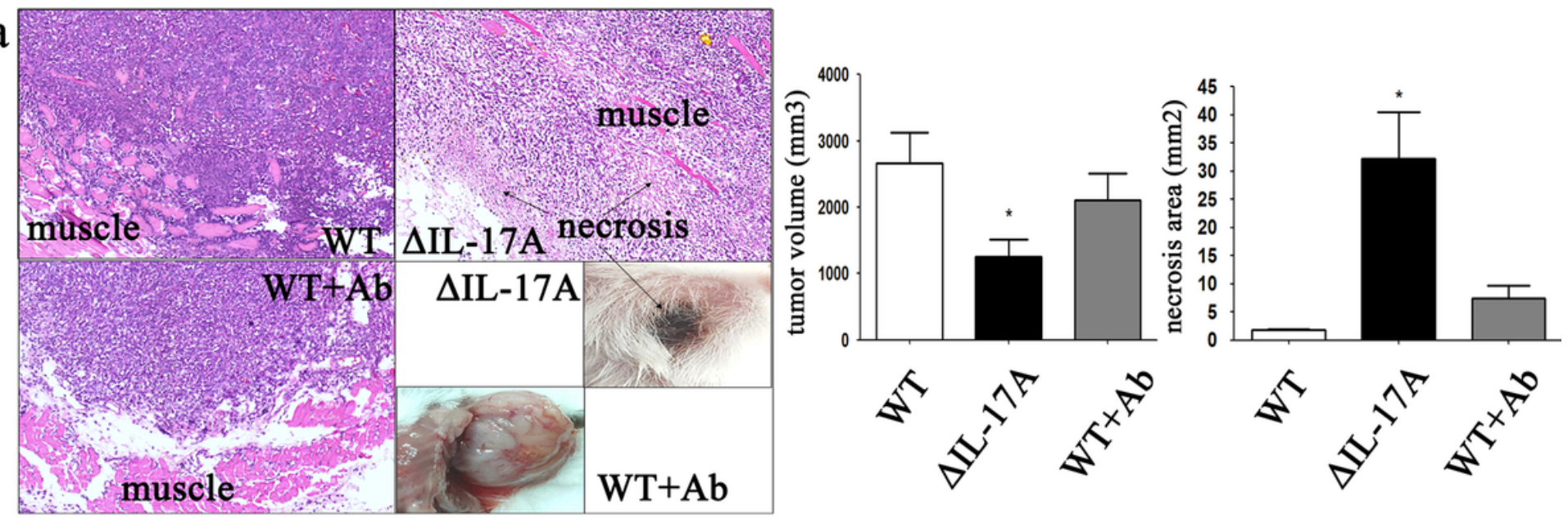

b
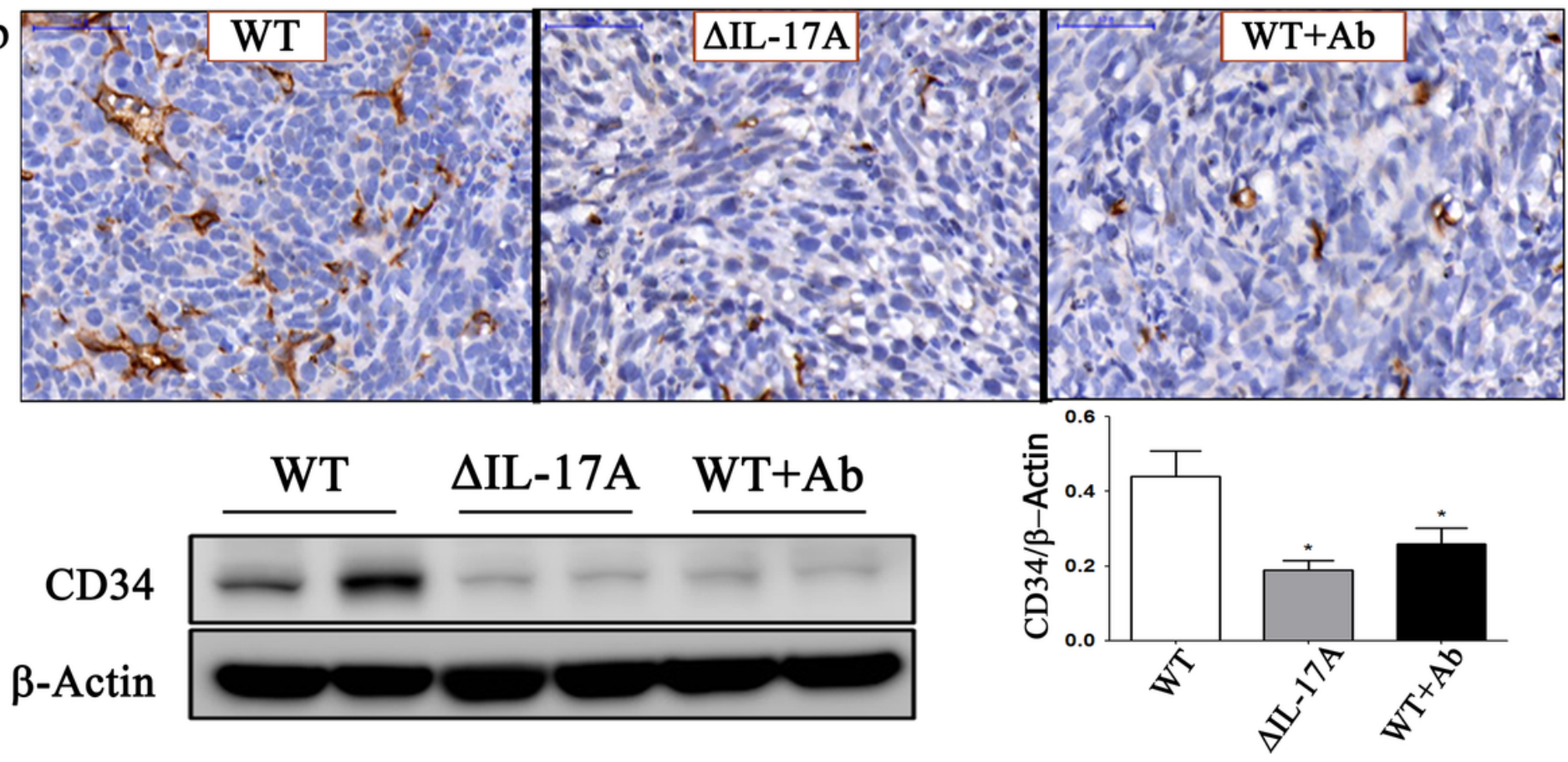

Figure 4

The role of IL-17A on in vivo implanted 4T1 cells in BALB/c mice. Three groups of 4T1 - bearing BALB/c mice were designed, namely, wild type (WT), IIL-17A, and WT + neutralizing IL-17 antibody (WT+Ab). After tumor cells were implanted. Neutralizing IL-17A antibody $(250 \mu \mathrm{g} / 100 \mu \mathrm{L})$ was injected, twice a week, intraperitoneally in WT+Ab group. After 4 weeks after tumor cells implantation, animals were sacrificed under adequate anesthetized. Tumor weight and necrosis area were photographed and measured (a). Immunohistochemistry and Western blot for CD34 expression was quantified (b). Bar indicated $50 \mu \mathrm{m}$. Asterisk indicates a p value $<0.05$, Mann-Whitney $U$ test. 
a
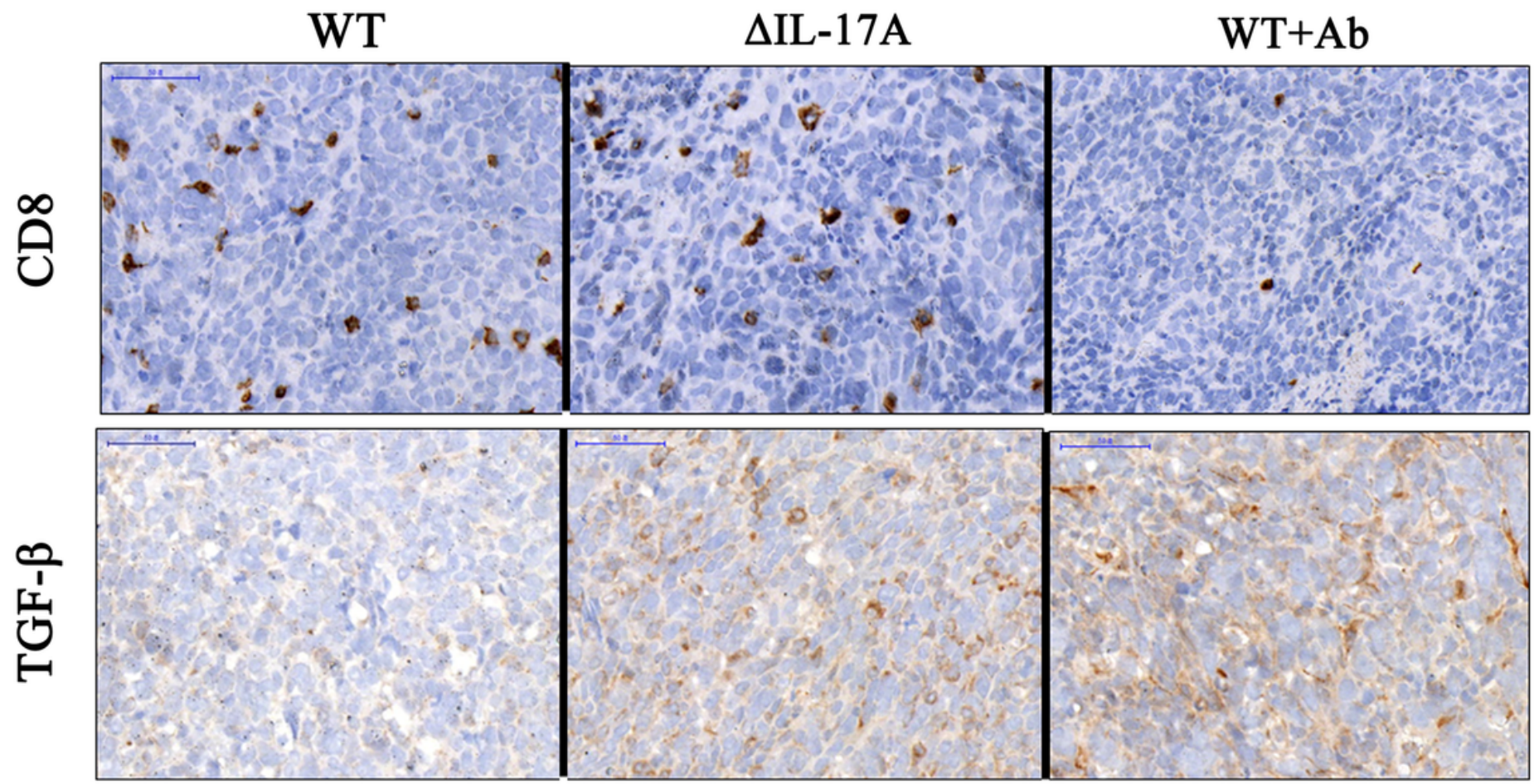

b
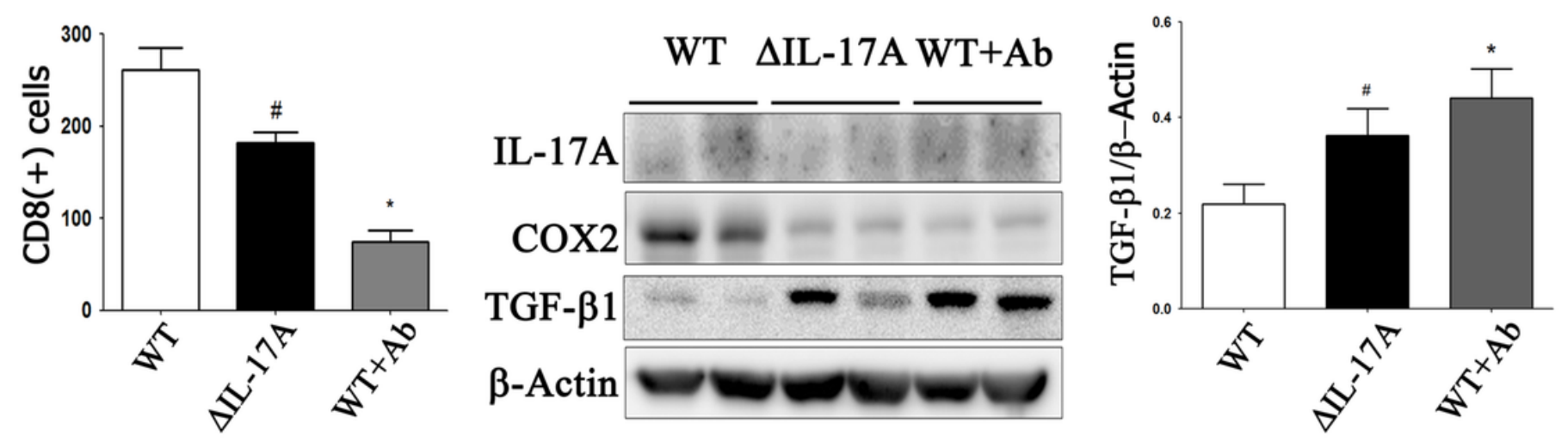

Figure 5

The role of IL-17A on CD8 and TGF- $₫ 14$ T1 cells-implanted BALB/c mice. Three groups of $4 \mathrm{~T} 1$-bearing BALB/c mice were designed, namely, wild type (WT), IIL-17A, and WT + neutralizing IL-17 antibody $(W T+A b)$. After tumor cells were implanted. Neutralizing IL-17A antibody $(250 \mu \mathrm{g} / 100 \mu \mathrm{L})$ was injected, twice a week, intraperitoneally in WT+Ab group. After 4 weeks after tumor cells implantation, animals were sacrificed under adequate anesthetized. CD8 and TGF- $\mathrm{1} 1$ expression were performed by immunohistochemistry (a) or/and Western blot (b). Bar indicated $50 \mu \mathrm{m}$. *, p value $<0.05$, Mann-Whitney U test, compared to the WT group; \#, p<0.05, Student t test, compared to the WT group. 


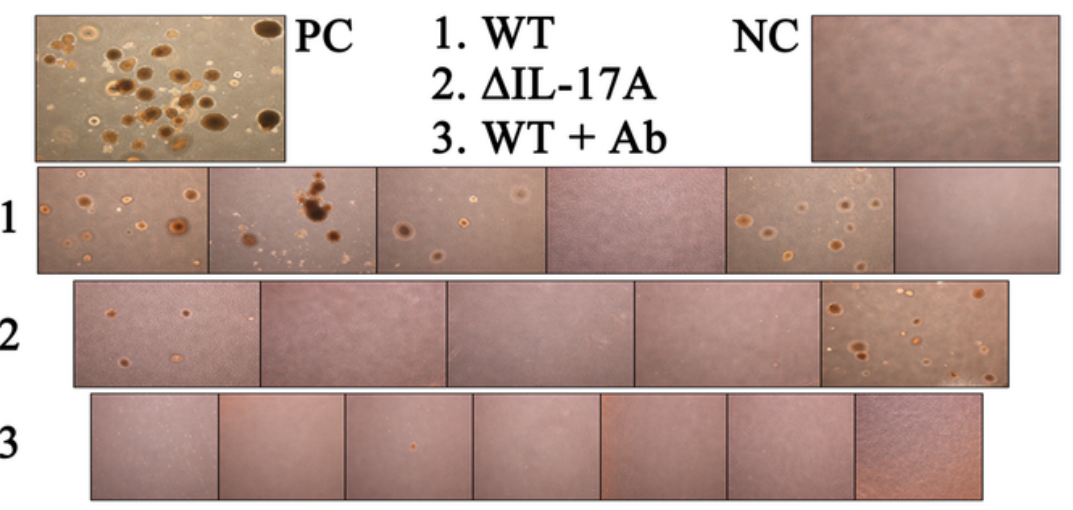

b

b
壳
这
产

WT $\quad \Delta$ IL-17A dead
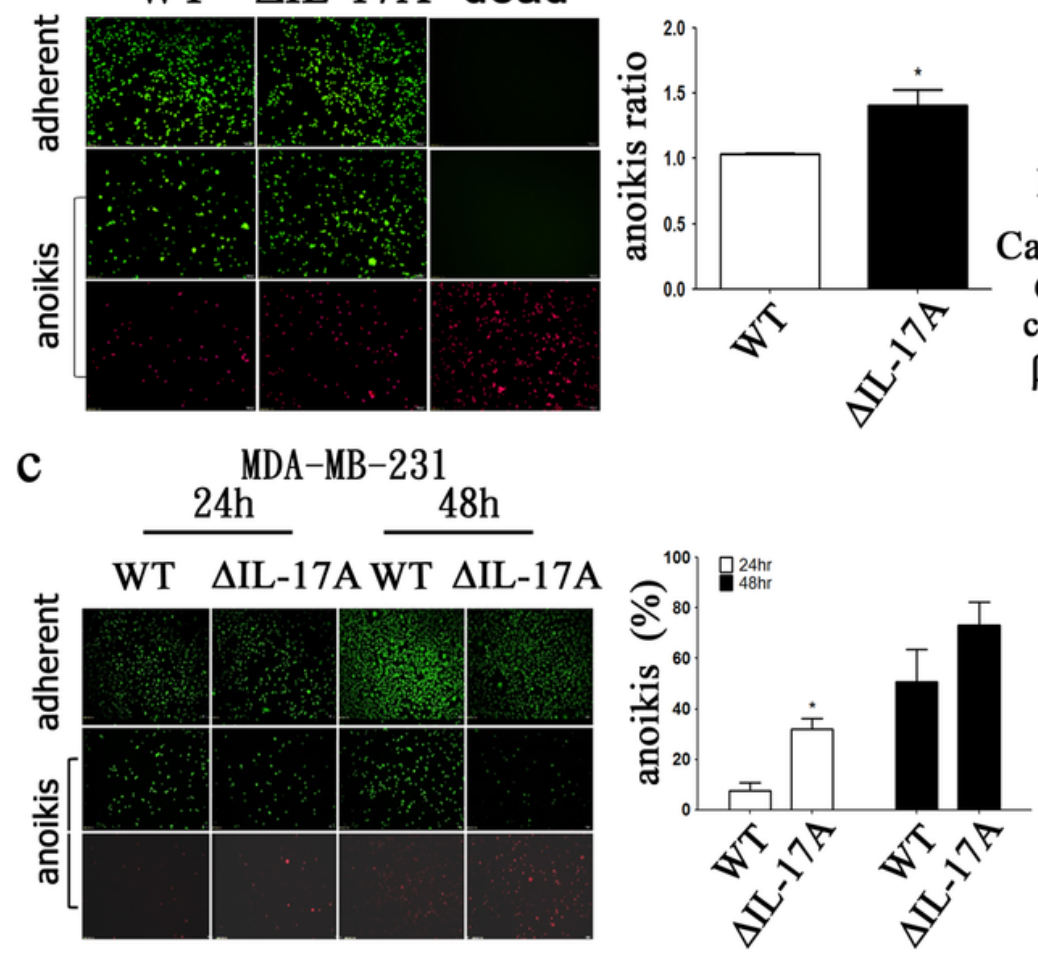

IL-17A

Caspase 3

Cleaved

caspase 3

$\beta$-Actin

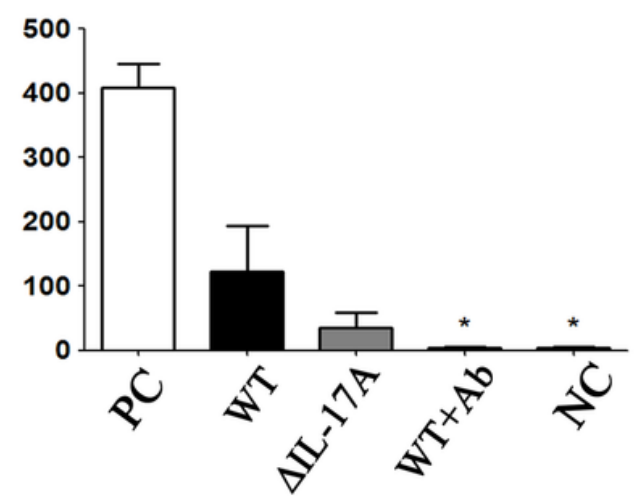

$0 \mathrm{~h} \quad 16 \mathrm{~h}$
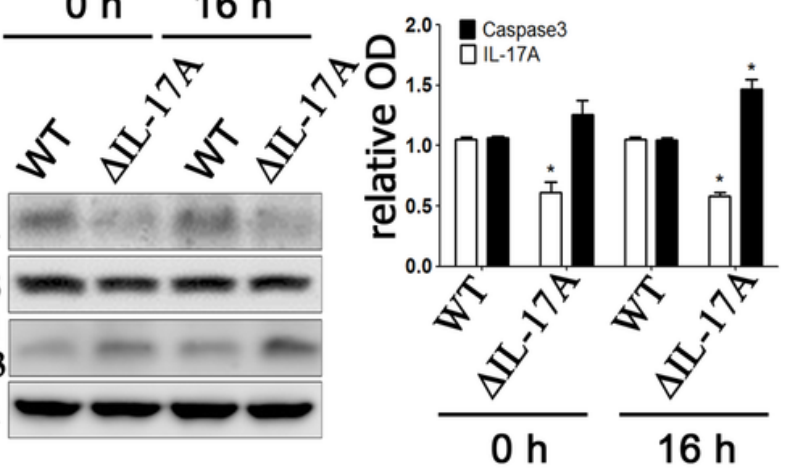

MDA-MB-468
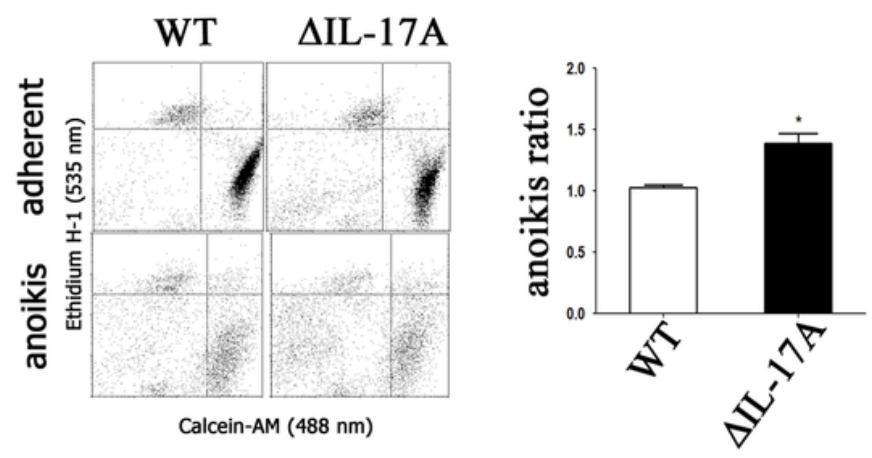

Figure 6

The role of IL-17A on circulating tumor cells (CTCs) and anoikis resistance in 4T1 cells-implanted BALB/C mice. Three groups of $4 \mathrm{~T} 1$ - bearing BALB/c mice were designed, namely, wild type (WT), ILL-17A, and WT + neutralizing IL-17 antibody (WT+Ab). After tumor cells were implanted. Neutralizing IL-17A antibody $(250 \mu \mathrm{g} / 100 \mu \mathrm{L})$ was injected, twice a week, intraperitoneally in WT+Ab group. After 4 weeks after tumor cells implantation, animals were sacrificed under adequate anesthetized. CTCs were isolated, subcultured, selected with 6-thioquanine $(60 \mathrm{LM})$, and ready for quantification with a 2-hydroxyethyl agarose colony assay (a). For anoikis assay, WT and IIL-17A 4T1 cells (b) or MDA-MB-231/MDA-MB-468 cells (c) were seeded onto adherent and anoikis (non-adherent) plate for $24 \mathrm{~h}$, followed by stained with Calcein$\mathrm{AM}$ and Ethidium homodimer-1 (live and dead stain) solutions and quantified by fluorescence microscopy (MDA-MB-231), flow cytometry (MDA-MB-468) or Western blot analysis as described in Methods. Relative optic density (O.D.) indicated IL-17A/ $\beta$-Actin or Caspase (cleaved/total) ratio. Asterisk indicated a $p$ value $<0.05$, compared to the WT group at the same time point (Mann-Whitney U test). 

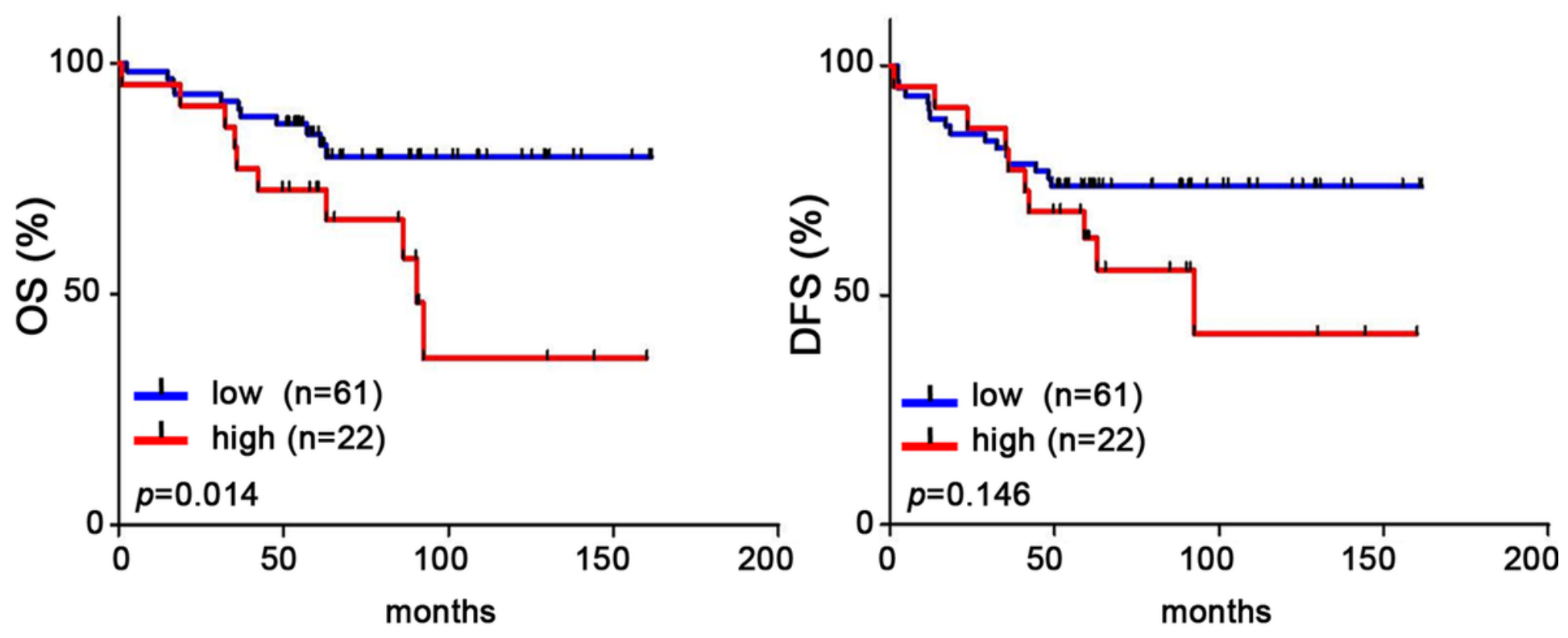

Figure 7

Clinicopathologic correlation between IL-17A expression and patients' prognosis. IL-17A protein expression by immunohistochemistry was correlated with patient survival, including overall survival (OS, a) and disease-free survival (DFS, b). The protein expression of IL-17A was semi-quantified and expressed as (0), $<10 \%,(1), 11-25 \%,(2), 26-50 \%$, and (3) $>50 \%$ of tumour cells. The MEGF11 expression level was defined as low $(\leq 60 \%, n=61)$ and high $(>60 \%, n=22)$. Asterisk indicated a $p$ value $<0.05$ by Kaplan-Meier survival analysis. 